\title{
Two Stage Data Fusion of Acoustic, Electric and Vibration Signals for Diagnosing Faults in Induction Motors
}

\author{
Anna Stief ${ }^{1}$, James R. Ottewill ${ }^{1}$, Michal Orkisz ${ }^{1}$, Jerzy Baranowski ${ }^{2}$ \\ ${ }^{1} A B B$ Corporate Research Center, ul. Starowislna 13a, 31-038, \\ Krakow, Poland, \\ ${ }^{2}$ Department of Automatics and Biomedical Engineering, Faculty of Electrical Engineering, \\ Automatics, Computer Science and Biomedical Engineering, AGH University of Science and \\ Technology, \\ Al. A. Mickiewicza 30, 30-059, Krakow, Poland \\ anna.stief@pl.abb.com
}

\begin{abstract}
The increasing demand for predictive maintenance is a main driver of the development of better fault diagnosis algorithms. Each condition monitoring approach has its own strengths and weaknesses; there is not a single technique that can diagnose all types of faults. As a result, it can be a challenge to find the root cause of a problem when only a single feature is monitored. There is also a greater risk of missed- or false-alarms. It has been shown that data fusion, combining multiple features, can improve the effectiveness of fault diagnosis. In recent work, a two-stage Bayesian inference approach, in which data is fused at both a local, or component, level, as well as at a global, or system-wide level has been shown to refine the diagnostic assessment of machinery comprised of a number of interacting components. In this paper, we show that the approach may also be applied to combine information from multiple, diverse condition monitoring systems. Acoustic, electric and vibration signals were measured from healthy and faulty induction motors, operating under normal and noisy working conditions. The proposed method was shown to increase the reliability of the health assessment of the induction motors, reducing the risk of missed and false alarms.
\end{abstract}

Index Terms-Bayesian inference; condition monitoring; data fusion; fault diagnosis; induction motors.

\section{INTRODUCTION}

The increasing demand for more accurate and reliable condition based- and predictive maintenance strategies is a main driver behind the development of improved fault diagnosis algorithms. Early stage fault diagnosis of industrial machinery is essential to avoid serious and costly failures. Each condition monitoring approach has its own strengths and weaknesses; there is not a single technique that can diagnose all types of faults. When only a single feature or a single signal type is monitored, finding the root cause of a problem can be challenging, increasing the potential risk of missed- or false-alarms.

Manuscript received 26 January, 2017; accepted 11 August, 2017.

This project has received funding from the European Union's Horizon 2020 research and innovation programme under the Marie SkłodowskaCurie grant agreement No 675215.
Recently several new condition monitoring methods have been developed, which fuse different types of signals in order to achieve better accuracy in identifying faults. It has been observed in multiple works (see for example [1]-[6]) that different signals are most informative for different faults: vibration signals are well suited for monitoring bearing faults [1], [2], current signals are good for detecting problems such as broken rotor bars or eccentricity relatedfaults [1], [3] and capacitor sensors may be used to measure the partial discharges that are indicative of stator winding insulation problems [1], [4].

For this reason, condition monitoring systems that fuse multiple signal types can be more accurate and robust at correctly identifying faults. Methods of fusing multiple signals for condition monitoring purposes have been reported by Safizadeh and Latifi [2], who fused vibration and load signals to diagnose bearing faults in induction motors, Khazee et al. [3], who combined vibration and acoustic signals to diagnose faults in gears, Yang and Kim [5], who fused vibration and current signals and Loutasa et al. [6], who combined vibration, acoustic and oil-debris signals to diagnose faults in gears. In each case, data fusion was shown to increase the accuracy of the system.

Bayesian inference is described in the literature as a suitable method for fault detection and fault classification in condition monitoring systems [7], [8]. Mehta et al. [9] used Naïve Bayesian classifiers to detect failures in a spindle bearing using ultrasonic and temperature data. Aydin et al. [10] used Bayesian classification to detect bearing faults in induction motors and classify motor conditions according to phase spaces. Recently Jaramillo et al. [11], presented a twostage Bayesian inference approach, in which data is fused at both a local, or component, level, as well as at a global, or system-wide level. The approach was shown to refine the diagnostic assessment of machinery comprised of a number of interacting components.

In this paper, we show that the two-stage Bayesian inference approach may also be applied to combine information from multiple, diverse condition monitoring 
systems. The fusion considers acoustic, electric and vibration signals from healthy and faulty induction motors operating under various load conditions. Signal features are extracted from the datasets and are analyzed using different statistical methods. Fault detection thresholds are developed experimentally using healthy motor datasets. In a primary, local step, features extracted from each type of signal are fused independently in order to obtain initial diagnoses of the health state of the system. A secondary global step, fuses the diagnoses from each of the signal types in order to obtain an overall diagnosis of the system. The proposed method is shown to be extremely reliable in providing accurate results about the health of the induction motors, thus reducing the risk of missed and false alarms.

The rest of the paper is organized as follows. In Section II the measurement data, which was used during the development of the algorithm, is described. In Section III the implemented data fusion method is introduced. Subsequently the results of fault diagnosis for the four induction motors are presented in Section IV. In Section $\mathrm{V}$ the results are discussed in greater detail and possible directions of future work are mentioned. Finally, in Section VI conclusions are given, highlighting the advantages and limitations of the method.

\section{DATA ACQUISITION}

Data was recorded from four $0,8 \mathrm{~kW}, 1400 \mathrm{rpm}$ induction motors (the SZJKe 14a), each having the same working parameters, but differing in terms of health state. Table I shows ratings of the motor SZJKE 14a. Experiments and development of fault detection and diagnostics methods using the same motors have been previously described in literature [12], [13]. The bearings of the motors were SKF type 6304 ZZ CXSQ.

TABLE I. RATINGS OF THE MOTOR SZJKE 14A.

\begin{tabular}{|c|c|}
\hline Parameter & Value \\
\hline Active power & $0,8[\mathrm{~kW}]$ \\
\hline Nominal voltage & $380[\mathrm{~V}]$ \\
\hline Nominal current & $2,2[\mathrm{~A}]$ \\
\hline Nominal power factor & 0,74 \\
\hline Rotor speed & $1400[\mathrm{rpm}]$ \\
\hline No load speed & $1497[\mathrm{rpm}]$ \\
\hline Winding connection & $\mathrm{Y}$ \\
\hline Number of poles per phase & 2 \\
\hline Nominal frequency & $50[\mathrm{~Hz}]$ \\
\hline Number of rotor bars & 22 \\
\hline Number of stator slots & 24 \\
\hline Rotor inertia & $0,0025\left[\mathrm{~kg} * \mathrm{~m}^{\wedge} 2\right]$ \\
\hline Coil number per phase & 4 \\
\hline Turn number of coil & 90 \\
\hline
\end{tabular}

The first motor was in a nominally healthy (without flaws) state (F1), the second had two broken rotor bars (F2), the third had an outer raceway defect in a bearing (F3) and the fourth had three broken rotor bars (F4). Micro flown [14] (acoustic particle velocity sensor), microphone, current, voltage and acceleration signals were measured. A rotating aluminium disk with eddy currents was the load, it was connected to each motor. Figure 1 shows the measurement set up.

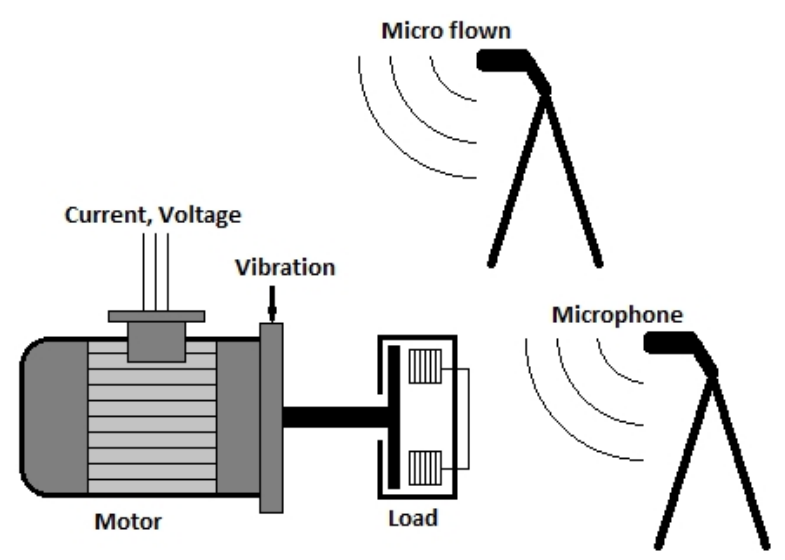

Fig. 1. Measurement set-up.

Acoustic signals were measured by three G.R.A.S. 46AE microphones and with a 3D Sound Intensity Micro flown probe, Model USP regular. The vibration signals were measured by 3 -axis PCB ICP accelerometers Model No. $356 \mathrm{~B} 18$ and 1-axis PCB ICP accelerometers Model No. 353B32. The voltages were measured by LV 25-P voltage trasnducers. The currents were measured by two LTS-6NP and two LEM HY 5-P current transducers. Data was collected using a 16 channel LMS Scadas Mobile System running LMS Test.Xpress 5A Software. The number of each measure signal type is shown below in Table II.

TABLE II. MEASURED SIGNALS.

\begin{tabular}{|c|c|c|}
\hline Type of signal & Unit & Number of signals \\
\hline Micro flown & {$[\mathrm{m} / \mathrm{s}]$} & 4 \\
\hline Microphone & {$[\mathrm{Pa}]$} & 3 \\
\hline Current & {$[\mathrm{A}]$} & 2 \\
\hline Vibration & {$\left[\mathrm{m} / \mathrm{s}^{2}\right]$} & 4 \\
\hline Voltage & {$[\mathrm{V}]$} & 3 \\
\hline
\end{tabular}

Measurements were acquired for each motor both with, and without additional noise from another separate motor. The sampling frequency was $51.2 \mathrm{kHz}$ and signals were recorded for 30 seconds, resulting in 1536000 data points. 5 different loads were considered for each motor. In total 37 viable datasets were obtained, distributed as follows: 10 F1, 10 - F2, 8 - F3, 9 - F4. A sliding window with 0,8 second overlap was applied to each dataset, which resulted in 146 windows for each measurement, and 5402 datasets in total.

\section{METHOD}

In this section we give a short description of the extracted features, the method used for setting alarm thresholds including how the load dependency of the threshold was taken into account, the Bayesian method applied and the proposed two-stage data fusion method. Computation were performed in MATLAB $\AA$ environment.

\section{A. Feature Extraction}

Both time and frequency domain features were extracted for each of the 16 signals. In the time domain the Root Mean Square (RMS), skewness, kurtosis, maximum Peak-to-Peak value and crest factor of each signal were calculated. In the frequency domain, the amplitude of the components at the 
first three harmonics of the supply frequency $(50 \mathrm{~Hz}$, $100 \mathrm{~Hz}, 150 \mathrm{~Hz})$, the first three harmonics of the rotation speed $(1 \mathrm{X}, 2 \mathrm{X}, 3 \mathrm{X})$, the ratio of the amplitude of the peaks at the second and third harmonic of rotation speed to the first harmonic $(2 \mathrm{X} / 1 \mathrm{X}, 3 \mathrm{X} / 1 \mathrm{X})$, and the amplitude of components at the sidebands of the supply frequency $(50 \mathrm{~Hz}$ $\pm 2 \times$ slip, $50 \mathrm{~Hz} \pm$ rotation speed) were calculated. Additionally, the frequency centre and spectrum area were also extracted.

In addition to the standard spectrum of the frequency domain signal, the spectrum of the envelope of the time domain signal was also analysed. The envelope of the signal is known to be a common method for identifying impulsive fault signatures, for example due to bearing faults [15], [16].

\section{B. Threshold Setting}

Once the features were extracted for each signal and dataset, the next step involved the determination of alarm thresholds for each feature. Threshold setting is one of the most crucial aspects of every condition monitoring system, as the accuracy of the end result is highly connected with choosing the appropriate thresholds.

The thresholds are calculated based on Kernel Density Estimation, which is described, for example in [11], [17], [18], as an accurate method when the exact distribution of the data is unknown. The kernel density estimators were constructed from healthy data for each feature and a $95 \%$ confidence interval (values of considered variables are only positive) was determined. The end of each confidence interval was set as a threshold, so that any feature value that exceeded the threshold would trigger an alarm.

\section{Load Dependency}

As many of the feature values are load dependent, improved results can be achieved if the thresholds are also load dependent. Data was recorded from the healthy motor operating under five different loads with no additional acoustic noise sources. These five datasets (five out of the 37 original dataset) were used to calculate five thresholds points for each feature. Fitting a first order polynomial to the five points allowed the threshold to be identified for any load (Fig. 2).

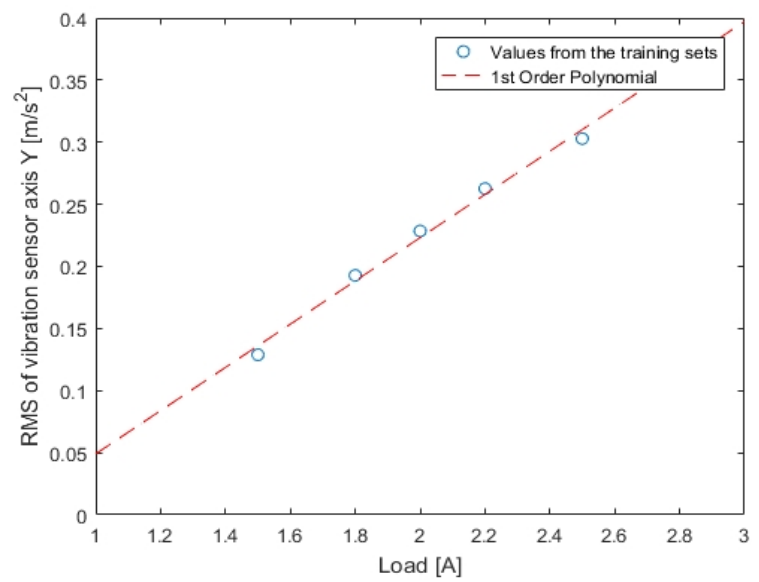

Fig. 2. Calculating the load dependent threshold.

\section{Bayesian Inference}

Recent work [11] proposed a two-stage Bayesian inference approach. The approach combines data fusion at both a local and a global level, or in other words at component and system-wide levels respectively. This method refines the diagnostic assessment of machinery comprised of a number of interacting components.

The Bayes-theorem is interpreted in the following way: the probability that a fault $F_{i}$ occurred in the system, given that a feature $y_{k}$ crosses its threshold is

$$
P\left(F_{i} \mid y_{k}\right)=\frac{P\left(y_{k} \mid F_{i}\right)}{P\left(y_{k}\right)} \cdot P\left(F_{i}\right) .
$$

If there are $M$ features $\mathbf{Y}=\left\{y_{1}, \ldots, y_{M}\right\}$ exceeding their respective thresholds the probability that fault $F_{i}$ occurred is

$$
P\left(F_{i} \mid Y\right)=\frac{P\left(Y \mid F_{i}\right)}{\sum_{j=1}^{M} P\left(y_{j} \mid F_{j}\right)} \cdot P\left(F_{i}\right) .
$$

This formalism is used for both the local stage and for the global stage.

\section{E. Local Fusion}

The local likelihood functions are formulated using four of the 37 original datasets, one from each fault case. The elements of the local likelihood functions represent the probability of a feature exceeding the threshold for a given fault case in the training sets. The prior probabilities are set to be equal for each fault case. The following algorithm calculates the posterior probabilities for each signal type: for each $i$ signal type we initialize $P^{0}(F)=\left\{p_{1}, \ldots, p_{i}, \ldots, p_{n}\right\}$, where $n$ is the number of fault cases and $p_{i}$ is the prior probability associated for fault case $F_{i}$. If $N$ features out of $M$ exceeded the threshold of signal type $i$ we update the prior probability by the equation:

$$
\left\{\begin{array}{l}
P_{k}^{\text {updated }}=\frac{P\left(y_{i, k} \mid F_{i}\right) \cdot P^{-}\left(F_{i}\right)}{\sum_{n=1}^{N} P\left(y_{i, k} \mid F_{i, n}\right) \cdot P^{-}\left(F_{i, n}\right)}, \\
P^{-}\left(F_{i}\right)=P_{k}^{\text {updated }} .
\end{array}\right.
$$

For the remaining $M-N$ feature which did not exceed the threshold of signal type $i$, we update the prior probability by the following equation:

$$
\left\{\begin{array}{l}
P_{k}^{\text {updated }}=\frac{\left(1-P\left(y_{i, k} \mid F_{i}\right)\right) \cdot P^{-}\left(F_{i}\right)}{\sum_{n=1}^{M-N}\left(1-P\left(y_{i, k} \mid F_{i, n}\right)\right) \cdot P^{-}\left(F_{i, n}\right)}, \\
P^{-}\left(F_{i}\right)=P_{k}^{\text {updated } .}
\end{array}\right.
$$

If the $P^{-}\left(F_{i}\right)$ probabilities are only updated when a feature exceeds its associated threshold, the probabilities will be biased towards predicting faults more often, even in the case of healthy systems, leading to an increase in false alarms. This problem is solved by (4) by updating $P^{-}\left(F_{i}\right)$ in case the threshold was not exceeded. 


\section{F. Global Fusion}

The global likelihood functions were constructed using 16 out of the 37 original datasets, four from each fault case, using datasets which were independent from the datasets used when constructing the local likelihood functions. The remaining 17 datasets were used as validation sets. After the local fusion was executed on all of the training sets, the posterior probabilities were calculated for each signal type, for each training set. The prediction of the state of the motor will be the index of the Maximum A Posteriori (MAP) probability in $P^{-}\left(F_{i}\right)$. Each signal type had a prediction for each training set, on the basis of which a probability matrix was built

$$
\mathbf{P}=\left[\begin{array}{ccc}
P\left(F_{1} \mid F_{1}\right) & \ldots & P\left(F_{n} \mid F_{1}\right) \\
\ldots & P\left(F_{i} \mid F_{j}\right) & \ldots \\
P\left(F_{1} \mid F_{n}\right) & \ldots & P\left(F_{n} \mid F_{n}\right)
\end{array}\right]
$$

The $P\left(F_{i} \mid F_{j}\right)$ probabilities were calculated on the basis of how many times the local stage predicted $F_{i}$ among the training sets, given that the actual fault case was $F_{j} . n$ is the number of fault cases. The matrix elements represent the "correctness" of a signal type predicting a fault case.

Once the local stage was executed for a validation set, 5 predictions were calculated based on the 5 indices of the Maximum A Posteriori (MAP) probabilities from the 5 signal types. The global likelihood function was built using the 5 probability matrixes of the 5 signal types, taking the MAP indexed column of each signal type from the corresponding probability matrix to the likelihood function. As in the local fusion step, the prior probabilities were set to be equal for each fault case. If the global likelihood function and the priori probabilities are known, (3) can be applied, allowing the final posterior probability to be calculated. The prediction of the state of the motor will be the index of the Maximum A Posteriori (MAP) probability of $P^{-}\left(F_{i}\right)$.

\section{RESULTS}

The two-stage data fusion algorithm has been applied to the validation sets and the results obtained have been compared both after the local fusion stage for each signal type separately, as well as after the global fusion stage. The fault cases are the following:

- F1: Healthy motor;

- F2: Motor with Two broken rotor bars;

- F3: Motor with Outer raceway defect in the bearing;

- F4: Motor with Three broken rotor bars.

The results of applying the proposed diagnostic procedure to the validation sets are presented via tables. In each table rows represent the actual health state of the motors under consideration, while columns represent the prediction output by the proposed method. The diagonal elements of the table represent proper diagnoses. For reference, the fault condition which is diagnosed most often for each fault case is highlighted.

\section{A. Results of Each Signal Type Separately}

The results from the local fusion stage are shown in Table III-Table VII. The probabilities in the tables represent how many predictions of the validation sets fall into a category $F_{i, j}$ divided by the number of validation sets of the actual motor in fault case $F_{i}$.

TABLE III. VIBRATION SIGNALS.

\begin{tabular}{|c|c|c|c|c|}
\hline \multicolumn{5}{|c|}{ Diagnosed Conditions } \\
\hline & F1 & F2 & F3 & F4 \\
\hline F1 & 0,71 & 0,00 & 0,00 & 0,29 \\
\hline F2 & 0,00 & 1,00 & 0,00 & 0,00 \\
\hline F3 & 0,00 & 0,00 & 0,94 & 0,06 \\
\hline F4 & 0,00 & 0,00 & 0,00 & 1,00 \\
\hline
\end{tabular}

The vibration signals are very good at distinguishing between faults in the motors, however they are only $71 \%$ accurate at recognizing healthy cases. In $29 \%$ of the investigated cases the vibration signals falsely indicated a fault of three broken rotor bars.

TABLE IV. CURRENT SIGNALS

\begin{tabular}{|c|c|c|c|c|}
\hline \multicolumn{5}{|c|}{ Diagnosed Conditions } \\
\hline & F1 & F2 & F3 & F4 \\
\hline F1 & 0,63 & 0,00 & 0,00 & 0,37 \\
\hline F2 & 0,03 & 0,97 & 0,00 & 0,00 \\
\hline F3 & 0,00 & 0,00 & 0,66 & 0,34 \\
\hline F4 & 0,00 & 0,00 & 0,00 & 1,00 \\
\hline
\end{tabular}

The current signals are very accurate in recognizing faults connected to broken rotor bars, but are less accurate then acceleration signals when diagnosing the case of an outer raceway defect in the bearing. The accuracy of recognizing healthy cases are worse compared to acceleration signals, false alarms occur in $37 \%$ of the cases.

TABLE V. MICRO FLOWN SIGNALS.

\begin{tabular}{|c|c|c|c|c|}
\hline \multicolumn{5}{|c|}{ Diagnosed Conditions } \\
\hline & F1 & F2 & F3 & F4 \\
\hline F1 & 0,41 & 0,58 & 0,01 & 0,00 \\
\hline F2 & 0,00 & 1,00 & 0,00 & 0,00 \\
\hline F3 & 0,00 & 0,33 & 0,67 & 0,00 \\
\hline F4 & 0,00 & 0,20 & 0,00 & 0,80 \\
\hline
\end{tabular}

In the experiments conducted the micro flown signals were not successful at differentiating between healthy motors and motors with two broken rotor bars. This is because the micro flown signal was the most sensitive signal type for noise. In half of the validation sets used for testing, additional background noise was generated by a separate motor. This additional noise resulted in a number of feature values exceeding their threshold, even in the case of a healthy motor. However, the micro flown signal was shown to be adept at distinguishing cases of two broken rotor bars, correctly diagnosing $100 \%$ of the validation sets. For recognizing the three broken rotor bars fault this signal type proved to be less accurate than the acceleration and current signals, however it did achieve a higher accuracy in detecting outer raceway bearing defects than the current signals. 
TABLE VI. MICROPHONE SIGNALS.

\begin{tabular}{|c|c|c|c|c|}
\hline \multicolumn{5}{|c|}{ Diagnosed Conditions } \\
\hline & F1 & F2 & F3 & F4 \\
\hline F1 & 0,95 & 0,00 & 0,00 & 0,05 \\
\hline F2 & 0,34 & 0,63 & 0,00 & 0,03 \\
\hline F3 & 0,40 & 0,00 & 0,59 & 0,00 \\
\hline F4 & 0,07 & 0,00 & 0,00 & 0,93 \\
\hline
\end{tabular}

Microphone signals were the most accurate at recognizing the healthy motor, with only $5 \%$ ratio of false alarms. However, these signals also had a relatively high proportion of missed alarms for the test cases investigated, where the system diagnosed a healthy motor in case of a faulty motor.

TABLE VII. VOLTAGE SIGNALS.

\begin{tabular}{|c|c|c|c|c|}
\hline \multicolumn{5}{|c|}{ Diagnosed Conditions } \\
\hline & F1 & F2 & F3 & F4 \\
\hline F1 & 0,28 & 0,52 & 0,20 & 0,00 \\
\hline F2 & 0,00 & 0,37 & 0,62 & 0,01 \\
\hline F3 & 0,00 & 0,40 & 0,57 & 0,03 \\
\hline F4 & 0,00 & 0,00 & 0,79 & 0,21 \\
\hline
\end{tabular}

The voltage signals are the least informative out of the five different signal types. The local data fusion generates false alarms in $72 \%$ of the investigated cases for the healthy motor. Voltage signals were also proven to be inaccurate at distinguishing between the different fault cases.

\section{B. Results of the Fused Signals}

The results of applying the second stage of the two-stage data fusion of the acoustic, electric and vibration signals can be seen in Table VIII. All of the validation sets taken from the motor with broken rotor bars were correctly diagnosed by the system. In $95 \%$ of the cases with outer raceway bearing defect the fault was correctly diagnosed, however in $5 \%$ of the cases the algorithm predicted healthy motor; a result that in practice would be viewed as a missed alarm. The healthy motor is correctly recognized in $99 \%$ of the cases, with false alarms being generated in $1 \%$ of the validation sets.

TABLE VIII. FUSED SIGNALS.

\begin{tabular}{|c|c|c|c|c|}
\hline \multicolumn{5}{|c|}{ Diagnosed Conditions } \\
\hline & F1 & F2 & F3 & F4 \\
\hline F1 & 0,99 & 0,00 & 0,00 & 0,01 \\
\hline F2 & 0,00 & 1,00 & 0,00 & 0,00 \\
\hline F3 & 0,05 & 0,00 & 0,95 & 0,00 \\
\hline F4 & 0,00 & 0,00 & 0,00 & 1,00 \\
\hline
\end{tabular}

TABLE IX. RESULTS OF FUSED SIGNALS.

\begin{tabular}{|c|c|c|}
\hline & Faulty prediction & Healthy prediction \\
\hline Faulty motor & TP: $98,63 \%$ & FN: $1,37 \%$ \\
\hline Healthy motor & FP: $1,10 \%$ & TN: $98,90 \%$ \\
\hline
\end{tabular}

Table IX. presents the true positive (TP), false positive (FP), false negative (FN) and true negative (TN) ratios for the overall results of the validation tests, where motor F1 was considered as healthy and motors F2, F3, F4 were considered as faulty.

\section{DISCUSSION}

On the basis of the results obtained, it is possible to evaluate which signal types are best suited to monitor particular mechanical faults. After the first stage the microphone signals were the most successful in recognizing the healthy motor in $95 \%$ of the cases ( $5 \%$ false alarms), whilst the least successful were the voltage signals, detecting only $28 \%$ of the healthy cases ( $72 \%$ false alarms). The vibration and the micro flown signals were $100 \%$ successful in detecting the fault case of a motor with two broken rotor bars. Again the voltage signals proved to be the least successful at diagnosing this case, detecting only $37 \%$ of the cases correctly. With a $94 \%$ success ratio the vibration signals were the most successful in detecting outer raceway bearing defect, falsely detecting three broken rotor bars in only $6 \%$ of these cases. The other types of signals performed significantly worse than the vibration signals in detecting outer raceway bearing defect, with the current, micro flown, microphone and voltage signals achieving a success rate of $66 \%, 67 \%, 59 \%$ and $57 \%$, respectively. In the case of motors with three broken rotor bars, vibration and current signals both had a $100 \%$ rate of successful detection, while the voltage signals were the least successful again, correctly diagnosing the fault in only $21 \%$ of the cases.

According to the results, the voltage signals were proven to be the least accurate in detecting the mechanical faults. In the future omitting this signal from the analysis could improve the results significantly, as well as simplifying the measurement process. It is a somewhat intuitive result that voltage signals would be the least suited to diagnosing the mechanical faults which were the primary focus of the investigation. Readers should note that if other fault types were to be considered (e.g. electrical faults such as winding short circuits) it might be anticipated that voltage signals would perform better as a fault indicator, in which case the signal type would again be a valid inclusion in the two stage data fusion process.

There are various other ways in which the accuracy of the method could potentially be improved. The load dependency model could also be extended in the future for more general cases, allowing alarm threshold values to be better approximated, and hence reducing the likelihood of false and missed alarms. Additionally, it is currently assumed that all health states are equally likely, whereas in practice this is unlikely to be the case. Improvement might be obtained by calculating a priori probabilities on the basis of historical fleet failure data.

\section{CONCLUSIONS}

In this paper we have shown that the fusion of acoustic, electric and vibration signals utilizing a newly-proposed two-stage Bayesian inference approach can significantly improve the accuracy of diagnosing faults in induction motors. The method was validated using an experimental system with acoustic, electric and vibration signals being measured from healthy and faulty induction motors.

The data fusion method is conducted in two stages. In the 
first stage, features extracted from each type of signal are fused independently in order to obtain initial diagnoses of the health state of the system. After this stage, the number of false alarms ranged from $5 \%$ of the total number of test cases for the microphone signals, up to $72 \%$ of the total number of test cases when considering voltage signals. In the second global fusion step, the output diagnoses obtained from the first fusion stages for each of the signal types are fused in order to obtain an overall diagnosis of the system. Applying this second stage allowed the number of false alarms to be reduced to $1 \%$ of the total number of test cases. Furthermore, the two-stage approach was proven to increase the accuracy of diagnosis with missed alarms only being observed in the case of bearing outer raceway fault ( $5 \%$ of tested cases).

A limitation of this approach is that, in its present form, the method is only suitable for steady state signals and does not take into account the severity of a feature exceeding the threshold. Furthermore, as a data-driven method, the accuracy of the likelihood functions used in the approach will be dependent on the availability of large quantities of comparable measurement data.

The results obtained indicated that the proposed method can increase the reliability and efficiency of fault detection. Furthermore the method provides a structured approach for comparing the performance of different signal types in diagnosing different fault modes.

\section{ACKNOWLEDGMENT}

The authors are thankful for the following people who carried out the measurement campaign at the Faculty of Electrical and Computer Engineering of Cracow University of Technology: Dr. Maciej Sułowicz, Dr. Konrad Weinreb, Dr. Janusz Petryna, Arkadiusz Dziechciarz.

\section{REFERENCES}

[1] S. Nandi, H. A. Toliyat, X. D. Li, "Condition monitoring and fault diagnosis of electrical motors-a review", IEEE Trans. Energy Conversion, 2005, vol. 20, no. 4, pp. 719-729. [Online]. Available: http://dx.doi.org/10.1109/TEC.2005.847955

[2] M. Safizadeh, S. Latifi, "Using multi-sensor data fusion for vibration fault diagnosis of rolling element bearings by accelerometer and load cell”, Information Fusion, vol. 8, pp. 1-8, 2014. [Online]. Available: http://dx.doi.org/10.1016/j.inffus.2013.10.002

[3] M. Khazaee, H. Ahmadi, M. Omid, A. Moosavian, M. Khazaee, "Classifier fusion of vibration and acoustic signals for fault diagnosis and classification of planetary gears based on Dempster-Shafer evidence theory", in Proc. Institution of Mechanical Engineers, Part E: Journal of Process Mechanical Engineering, vol. 228, no. 1, pp. 21-32, 2014. [Online]. Available: http://dx.doi.org/10.1177/0954408912469902

[4] G. Dauksys, A. Jonaitis, S. Gudzius, A. Morkvenas, "Investigation of partial discharges at the high voltage electric motor bars", Elektronika ir Elektrotechnika, vol. 22, no. 2, pp. 9-12, 2016.
[Online]. Available: http://dx.doi.org/10.5755/j01.eie.22.2.6983

[5] B. S. Yang, K. J. Kim, "Application of Dempster-Shafer theory in fault diagnosis of induction motors using vibration and current signals", Mechanical Systems and Signal Processing, vol. 20, no. 2, pp. 403-420, 2006. [Online]. Available: http://dx.doi.org/10.1016/ j.ymssp.2004.10.010

[6] T. H. Loutasa, D. Rouliasa, E. Paulyb, V. Kostopoulos, "The combined use of vibration, acoustic emission and oil debris on-line monitoring towards a more effective condition monitoring of rotating machinery", Mechanical Systems and Signal Processing, vol. 25, no. 4, pp. 1339-1352, 2011. [Online]. Available: http://dx.doi.org/ 10.1016/j.ymssp.2010.11.007

[7] A. Heng, S. Zhang, A. C. C. Tan, J. Mathew, "Rotating machinery prognostics: State of the art, challenges and opportunities", Mechanical Systems and Signal Processing, vol. 23, no. 3, pp. 724739, 2009. [Online]. Available: http://dx.doi.org/10.1016/ j.ymssp.2008.06.009

[8] K. Tidriri, N. Chatti, S. Verron, T. Tiplica, "Bridging data-driven and model-based approaches for process fault diagnosis and health monitoring: A review of researches and future challenges", Annual Reviews in Control, vol. 42, pp. 63-81, 2016. [Online]. Available: http://dx.doi.org/10.1016/j.arcontrol.2016.09.008

[9] P. Mehta, A. Werner, L. Mears, "Condition based maintenancesystems integration and intelligence using Bayesian classification and sensor fusion", Journal of Intelligent Manufacturing, vol. 26, no. 2, pp. 331-346, 2015. [Online]. Available: http://dx.doi.org/10.1007/ s10845-013-0787-1

[10] I. Aydin, M. Karakose, E. Akin, "Combined intelligent methods based on wireless sensor networks for condition monitoring and fault diagnosis", Journal of Intelligent Manufacturing, vol. 26, no. 4, pp. 717-729, 2015. [Online]. Available: http://dx.doi.org/10.1007 /s10845-013-0829-8

[11] V. H. Jaramillo, J. R. Ottewill, R. Dudek, D. Lepiarczyk, P. Pawlik, "Condition monitoring of distributed systems using two-stage Bayesian inference data fusion", Mechanical Systems and Signal Processing, vol. 87, pp. 91-110, 2017. [Online]. Available: http://dx.doi.org/10.1016/j.ymssp.2016.10.004

[12] M. Orman, M. Orkisz, C. T. Pinto, "Parameter identification and slip estimation of induction machine", Mechanical Systems and Signal Processing, vol. 25, no. 4, pp. 1408-1416, 2011. [Online]. Available: http://dx.doi.org/10.1016/j.ymssp.2010.11.004

[13] A. Ukil, S. Chen, A. Andenna, "Detection of stator short circuit faults in three-phase induction motors using motor current zero crossing instants", Electric Power Systems Research, vol. 81, no. 4, pp. 10361044, 2011. [Online]. Available: http://dx.doi.org/10.1016/ j.epsr.2010.12.003

[14] H. E. De Bree, "The Microflown: An acoustic particle velocity sensor", Acoustics Australia, vol. 31.3, pp. 91-94, 2003.

[15] R. B. Randall, Vibration-Based Condition Monitoring: Industrial, Aerospace and Automotive Applications. Hoboken, New Jersey: John Wiley \& Sons, 2011,pp. 47-52. [Online]. Available: https://doi.org/ $10.1002 / 9780470977668$

[16] M. Riera-Guasp, J. A. Antonino-Daviu, G. A. Capolino, "Advances in electrical machine, power electronic, and drive condition monitoring and fault detection: state of the art", IEEE Trans. Industrial Informatics, vol. 62, no. 3, pp. 1746-1759, 2015. [Online]. Available: http://dx.doi.org/10.1109/TIE.2014.2375853

[17] Q. Chen, P. Goulding, D. Sandoz, R. Wynne, "The application of kernel density estimates to condition monitoring for process industries", in Proc. American Control Conf., vol. 6, pp. 3312-3316, 1998. [Online]. Available: https://doi.org/10.1109/ACC.1998.703187

[18] P. E. P. Odiowei, Y. Cao, "Nonlinear dynamic process monitoring using canonical variate analysis and kernel density estimations", IEEE Trans. Industrial Informatics, vol. 6, no. 1, pp. 36-45, 2010. [Online]. Available: http://dx.doi.org/10.1109/TII.2009.2032654 